\title{
Pupil size as a window on neural substrates of cognition
}

\author{
Siddhartha Joshi and Joshua I. Gold \\ Department of Neuroscience, University of Pennsylvania, Philadelphia, PA 19104
}

\section{CONTACT:}

Siddhartha Joshi: sidjoshi@pennmedicine.upenn.edu

\section{Keywords:}

cognition, autonomic nervous system, brain state, arousal, locus coeruleus, superior colliculus 


\begin{abstract}
Pupil modulations provide a non-invasive window onto how the brain performs many cognitive tasks. Here we review recent work that has begun to identify specific neural underpinnings of these cognitively driven pupil signals, including key roles for: 1) the superior colliculus (SC), which mediates responses to salient stimuli that evoke orienting responses; and 2) the locus coeruleus (LC), the source of a major neuromodulator (norepinephrine) that mediates arousal and cognition. We discuss how these findings can inform the interpretation of pupil measurements in terms of specific neural operations involving the SC and LC. We also highlight caveats, open questions, and key future experiments for improving the interpretation of pupil measurements in terms of the underlying neural dynamics throughout the brain.
\end{abstract}




\section{Changes in pupil size encode cognitive variables}

Pupil size changes continuously in response to changes in ambient light levels to regulate the amount of light entering the eye, which is a well-known autonomic phenomenon called the pupillary light reflex (PLR, $[1,2])$. In addition to the PLR, pupil size can also change under steady lighting conditions in response to a range of cognitively relevant factors including changes in stimulus salience, effort, and internal state [3-5]. For example, as early as 1929 it was noted that: "deep emotions of pleasure as well as fear are commonly accompanied by pupillary dilation" $[3,4]$. Later it was recognized that these pupil modulations may provide a window into at least some aspects of the inner workings of the brain that are otherwise typically inaccessible; e.g., "the pupils... register directly certain activities of the nervous system, including, but not restricted to, the effects of visual stimulation" [4]. This idea, combined with the relative ease with which pupil size can be measured, has led to a growing number of studies that use pupil measurements to draw conclusions about specific features of neural processing that contribute to perception, decision-making, and other aspects of cognition. However, these approaches

also raise an important question: What, exactly, can we infer about brain function from measurements of pupil size?

The goal of this article is to review our current state of understanding of this "inverse problem." That is, given what we know about neural mechanisms underlying cognition that also cause non-luminance-mediated changes in pupil diameter, what can we infer about those mechanisms from measurements of pupil alone? We start with the forward problem and describe studies that have identified pupil modulations that result from, or are at least correlated with, activation of particular neural systems. We focus on two systems that relay relevant top-down, luminanceindependent signals onto autonomic circuits that directly control pupil diameter: the superior colliculus (specifically the intermediate layers, or $\mathrm{SC}_{\mathrm{i}}$ ) in the midbrain and the locus coeruleus (LC) in the pontine brainstem. We emphasize the relatively small number of studies that have made direct, simultaneous measurements of neural activity in these systems and pupil diameter. We then consider directly the inverse problem in terms of inferring neural activity in these systems from two common measures of pupil size used in a broad range of cognitive studies: baseline and event-driven changes in pupil size. We highlight both the strengths and weaknesses of our current state of knowledge and conclude with a discussion of possible future directions.

\section{The forward problem: measuring brain activity in relation to pupil size}

Autonomic circuits that drive pupil dilation and constriction are located at the level of the spinal cord and midbrain (Figure 1). These circuits operate in response to changes in light levels and are also influenced by central descending inputs that drive pupil size changes under steady illumination (Box 1). Many of these inputs converge through two nuclei (Box 2): 1) the SC, which is a specialized motor nucleus that integrates a wide range of sensory and associative signals to generate commands for orienting and moving the eyes [33-35]; and 2) the LC, which also receives a range of inputs from a number of brain regions [36-40] and is the primary source of central NE, via which it regulates global arousal [41-43]. Accordingly, non-luminance-mediated changes in pupil size are often interpreted in terms of activation of these two nuclei and the larger networks in which they operate $[44,45]$. Here we review the compelling, albeit still limited, evidence that goes beyond these anatomical findings and shows more direct, functional relationships between $\mathrm{SC}_{\mathrm{i}}$ and $\mathrm{LC}$ neural activity and pupil size. 


\section{Evidence for functional relationships between $\mathrm{SC}_{\mathrm{i}}$ activity and pupil size}

The evidence for functional relationships between SC activation and pupil size is solid but relatively sparse. One prominent pair of studies examined these relationships in the context of the orienting reflex, which involves shifts of gaze and attention that are mediated in part by the $\mathrm{SC}$. The first showed that pupil dilations are evoked by electrical microstimulation in the $\mathrm{SC}_{i}$, which receives sensory, motor, and cognitive information from cortex, but not in more superficial layers of the SC, which receives predominantly visual information [53]. These microstimulation effects, which tended to be larger in low light conditions, were interpreted in terms of a possible role in enhancing visual sensitivity or attention. The second, follow-up study showed that both visual and auditory stimuli evoke pupil responses and changes in neural activity in $\mathrm{SC}_{\mathrm{i}}$ that are modulated in a similar manner by contrast-based saliency, which is a key driver of shifts in gaze and attention [56]. Together these studies provide the strongest evidence that stimulus-driven pupil responses that occur in tandem with overt or covert orienting responses reflect functions of the $\mathrm{SC}_{\mathrm{i}}$.

These results are supported by another study that reported relationships between pupil diameter and both single-unit spiking and electrical microstimulation in the $\mathrm{SC}_{i}[54]$. In that study, individual $\mathrm{SC}_{i}$ spikes were reliably associated with transient dilations that, in principle, could result from the direct projections from the $S C_{i}$ to brainstem nuclei that control pupil diameter (see Figure 1 and Box 2). However, higher $S C_{i}$ spiking activity measured over longer timescales (up to several seconds) was reliably associated with more constricted pupils. These results suggest the possibility of multiple pathways driving relationships between $\mathrm{SC}_{\mathrm{i}}$ and pupil under different conditions and different timescales. The functional roles of these pathways are currently being investigated and are likely linked with the established role of $\mathrm{SC}_{\mathrm{i}}$ in conveying signals related to stimulus salience and spatial attention $[55,56]$.

\section{Evidence for functional relationships between LC activity and pupil size}

The evidence for functional relationships between LC activation and pupil size is more extensive but also still incomplete. Indirect evidence for these relationships first came from measurements of global arousal using electroencephalography (EEG), which was found to covary with pupil size and separately with LC activity $[68,108-110,120]$. More recently, pupil size has been related to event-related potentials (ERPs) also measured from the scalp, specifically to the P3 component of the ERP in humans performing an auditory oddball task [69]. The P3 is thought to reflect activation of the LC-NE system, although the current evidence for this relationship is indirect and somewhat contested [70]. Other indirect evidence for a pupil-LC link has come from studies that used pharmacological approaches to perturb the LC-NE system, resulting in pupil changes $[65,146]$. Together, these studies provided enough indirect evidence that even before extensive measurements of relationships between pupil size and LC neural activity were obtained, the two were generally assumed to covary. Consequently, pupil measurements have long been interpreted in terms of LC neural activation [44,52,65,69,70,142].

Recent studies have begun to provide more direct evidence in the form of simultaneous measurements of pupil and LC. In particular, several studies have shown that pupil size tends to covary with LC neural firing rates measured in the same epoch, under mainly passive task conditions $[54,66,67]$. First, fast dilations of the pupil were associated with phasic activity in LCNE axons in the cortex of awake mice [67]. Second, LC single-neuron activity was shown to covary with pupil size over long (over several seconds) and short (associated with single LC spikes) timescales in monkeys performing a basic fixation task. Moreover, electrical microstimulation of LC in these monkeys reliably evoked pupil dilations [54]. Third, changes in pupil size that covary with BOLD activity were localized to LC in a pioneering imaging study of humans performing an oddball task [68]. Fourth, pupil dilation correlated with LC neuron firing 
rate in monkeys performing a task designed to assess neuromodulatory effects on effort and reward processing around the time of goal-directed actions, although that study did not describe the timing of pupil changes relative to LC neuron firing [66].

\section{Caveats and open questions for interpreting pupil diameter in terms of neural activity}

The findings summarized above provide compelling support for interpreting pupil diameter in terms of $\mathrm{SC}_{\mathrm{i}}$ and LC activation patterns. However, there are presently no direct simultaneous measurements of neural activity in both $\mathrm{SC}_{\mathrm{i}}$ and $\mathrm{LC}$ along with measurements of pupil diameter. Such measurements are needed to better delineate similarities and differences in how these two different brain areas relate to pupil, under different cognitive conditions. Other caveats and open questions also remain, including the following:

Do pupil changes reflect neural activity in predominantly one brain region, or many? Both the $\mathrm{SC}_{\mathrm{i}}$ and LC are interconnected with many brain regions. The SC receives cortical and subcortical inputs and projects to other nuclei, particularly to nuclei that control gaze and to specific visuo-motor cortical regions [34,46,47,138-141]. The LC receives cortical and subcortical inputs and projects broadly throughout the brain [36-43]. Thus, in principle any covariation between pupil size and neural activity in the $\mathrm{SC}_{i}$ and/or the LC likely also reflects activity in many other brain regions with which they interact. If and how pupil diameter depends on the specific, context-dependent forms of these interactions is not well understood.

Understanding these interactive effects requires distinguishing cause (that is, is neural activity in a particular brain area causing changes in $\mathrm{SC}_{i}$ or $\mathrm{LC}$ that are associated with pupil changes?) from effect (conversely, are changes in neural activity in a particular brain area a result of $\mathrm{SC}_{i}$ or LC activation that is associated with pupil changes?). For example, pupil size was shown to covary with fluctuations in the depolarization, hyperpolarization, and slow oscillations of singleneuron membrane potentials and changes in correlated population activity and sensory responses of neurons in sensory cortex [71-73]. Because these neural dynamics were affected diffusely, these results were interpreted as an effect of NE release on cortex and not the cause of LC activation. Another recent study used the relative timing of relationships between pupil changes and neural activity changes in the LC and a number of other subcortical and cortical brain areas to arrive at a similar conclusion: a primary association between pupil and LC can correspond to secondary associations between pupil and targets of LC-NE neuromodulation [54]. Testing these ideas directly and more generally distinguishing causal influences on $\mathrm{SC}_{\mathrm{i}}$ and LC from effects of changes in their activity patterns will require, for example, both recording and manipulating activity in these brain areas while recording the activity of their neural targets and pupil diameter.

Do pupil changes reflect the activity of all LC neurons, or are they sensitive to the anatomical and functional heterogeneity of the LC? Early descriptions of the LC emphasized its functional and anatomical homogeneity [74,75,95,96]. The persistence of these views has led to interpretations of pupil changes in terms of overall LC activation. However, recent studies have begun to identify anatomical and functional heterogeneity within the LC and in its connectivity patterns $[76-84,97]$. These findings suggest that there might also be heterogeneity in task- and neuron-specific relationships between LC activity and pupil diameter. In support of this idea, studies comparing LC activity to pupil size have shown neuron-specific differences in the strength and direction of relationships between the two [54, 82]. Further work is needed to identify the structural and functional characteristics of LC neurons that are responsible for these differences and if and how these relationships depend on the task context.

Do pupil changes reflect activity in multiple neuromodulatory systems? The basal forebrainacetylcholine (BF-ACh) system, which is associated with modulation of sensory as well as 
cognitive functions, including attention, has widespread projections throughout the brain [85-88]. Pupillometry has been used to index changes in the BF-ACh system in relation to attention, similar to the use of pupillometry as an index of LC-NE linked global arousal $[44,68-70,89-$ 91,102,104,114]. As in the case of the LC-NE system, direct, quantitative evidence for correlations between BF-ACh activation and changes in pupil size has come only very recently $[67,92]$. However, the circuit bases of these correlations remain unknown. One possibility involves NE effects via LC projections to the BF that can depolarize cholinergic neurons via $\alpha 1$ adrenergic receptors $[86,93,94]$. A direct comparison of the two systems provided indirect support for this mechanism by showing that LC-linked pupil changes preceded ACh-linked pupil changes by $\sim 0.5 \mathrm{~s}$ [67].

\section{The "inverse problem": Inferring brain activity from measurements of pupil size}

The relative ease of conducting pupillometry experiments compared to invasive brain measurements, along with a growing understanding of the neural substrates of cognitively mediated pupil modulations, have led to a rapid growth in the number of studies that use pupillometry to study a broad range of cognitive processes. Here we review those studies in terms of how they have interpreted two kinds of pupil measurements: 1) baseline pupil values that persist over relatively long timescales and are typically linked with overall brain state and its effects on arousal and attention; and 2) transient pupil responses to specific, task-relevant events. These measurements have a commonly cited but somewhat tenuous relationship to tonic and phasic firing modes of LC neurons, respectively (see the "Caveats" section, below), and under certain conditions may also reflect activity in the $\mathrm{SC}_{\mathrm{i}}$ and elsewhere.

\section{Brain activation inferred from baseline pupil size}

In many human behavioral experiments, baseline pupil size is measured during passive task epochs before the presentation of task-related stimuli. These baseline pupil values, which have been identified in the context of task-related changes in exploration, belief uncertainty, engagement, effort (e.g., memory load), and other factors, are often interpreted in terms of cognitively relevant functions of the LC [44,102,106,112,114,134]. For example, an early evaluation of the relationship between pupil size and memory load used a task in which subjects had to hear and then immediately recall three to seven digits in each trial. Baseline pupil size was found to increase with the number of digits that had to be held in memory [134]. Such links between pupil size and working memory are now thought to be mediated, at least partly, by the LC-NE system [136].

Another study involved a systematic exploration of the relationship between pupil size and the cognitive control of adaptive behavior [44]. Building on an earlier study in behaving monkeys that linked the activity of individual LC neurons with performance on a visual oddball task [129], this study found a similar relationship between pupil diameter and performance on an auditory oddball task. This study further identified modulations of baseline pupil diameter in terms of changes in costs and rewards on an auditory discrimination task, and in terms of diminishing utility and decisions to transition from an exploit to an explore state [44]. The authors interpreted these baseline pupil modulations in terms of a previously proposed theory about LC-mediated regulation of adaptive state changes in the brain [96].

Baseline pupil size might also, under certain conditions, reflect neural activity in $\mathrm{SC}_{\mathrm{i}}$, via its role in spatial attention that is modulated, in part, by the BF-ACh system [55-58,132,147]. The activity of ACh axons imaged in awake mouse visual cortex has been shown to covary with pupil size over relatively long timescales associated with changes in locomotor state [67]. Pharmacological studies have also suggested a relationship between spatial attention, BF-ACh activity, and baseline pupil size, although such ACh-linked effects could in principle result from 
direct action of these pharmacological agents on the sphincter and dilator pupillae $[116,117]$. Direct, simultaneous measurements are still needed to assess whether and how the cholinergic BF and pupil size are related under particular cognitive conditions. It also will be useful to design tasks that manipulate factors that selectively engage the $L C, S_{i}$, or $B F$ to determine their possibly distinct relationships to baseline pupil diameter.

\section{Brain activation inferred from transient pupil responses}

A host of cognitive factors have been shown to drive brief pupil dilations that are also linked with direct and indirect measures of LC and $\mathrm{SC}_{\mathrm{i}}$ activation. In human subjects, stimulus probability, including the presence of an unexpected stimulus or the absence of an expected stimulus, is directly related to the amplitude of the P3 component of the ERP and inversely linked to transient pupil dilation, both of which have been associated with LC activation [119-121,137]. For example, one study manipulated the probability of auditory targets and showed that the evoked pupil response was sensitive to the surprise value of the event, even when the event was the absence of a stimulus [119]. Another study showed that the amplitude of the P3 was inversely related to the probability of occurrence of task-relevant stimuli [137].

Task-relevant, evoked pupil dilations have also been associated with stimulus salience, stimulus value, attentive state, prior expectation, estimation error, decision biases, and changes in task utility $[4,102,104,106,122,123,126]$. Because many of these factors can also drive transient increases in LC neuron firing rates [96,127-129], transient pupil dilations are often interpreted in terms of such phasic LC firing. In addition, some of these factors including stimulus salience are associated with orienting responses and thus are also likely associated with $\mathrm{SC}_{\mathrm{i}}$ activation [45,53,127-130]. Consistent with this idea, when neural activity in $\mathrm{SC}_{\mathrm{i}}$ was manipulated using electrical microstimulation and pharmacology, transient changes in pupil size were found to be modulated by stimulus features at an attended location [55].

However, interpreting task-driven changes in pupil diameter in terms of neural activity patterns in $\mathrm{LC}, \mathrm{SC}_{\mathrm{i}}$, or both, is certainly an oversimplification and ignores the specific contributions of higher brain areas that are responsible for these task-specific effects. The relevant computations are likely implemented in a number of brain regions, including parts of the prefrontal cortex, particularly the anterior cingulate cortex (ACC), a part of medial prefrontal cortex (mPFC) that is one of the main descending cortical inputs to LC $[96,111,115,118,124$ $126,131,133]$. For example, ACC neurons can encode surprise, conflict, and other forms of uncertainty that also can modulate pupil diameter [124,135,143,144], including in one case measured simultaneously [145]. ACC neural activity has also been shown to covary with pupil modulations under passive viewing conditions, over both short (single spike) and longer (several seconds) timescales [54]. Therefore, although it is possible to infer transient LC and $\mathrm{SC}_{\mathrm{i}}$ activation from task-relevant, transient pupil dilations, more work is needed to determine the other brain regions that are also active under the same conditions and might be responsible, in part, for top-down pupil modulations. Recent studies have begun to identify anatomically distinct cortical circuits that are affected by, and can affect, the LC under different conditions [81, 82]. It remains to be determined if and how this kind of structural and functional heterogeneity of LCrelated information processing can be inferred from different characteristics of the pupil response.

\section{Caveats and open questions for interpreting baseline and evoked pupil measures}

Despite the advances in our understanding of how to interpret baseline and pupil measures in terms of the underlying neural dynamics, a number of caveats and open questions remain, including the following: 
How to distinguish task-relevant from task-irrelevant changes in autonomic tone? During goaldirected behaviors, the LC is driven by descending, task-relevant signals from cortex, and its activation can drive changes in baseline pupil size that reflect such signals. However, taskirrelevant, autonomic modulation of LC activity can also result in the release of NE with similar effects on pupil [31]. Can LC-linked pupil fluctuations resulting from these distinct regimes be distinguished from each other? One possible answer is to distinguish passive versus active behavioral epochs, using the former to characterize autonomic-related fluctuations that can be factored out when analyzing task-related fluctuations in the latter. Operationally, such baseline autonomic tone can be estimated using passive fixation periods preceding presentation of taskrelated stimuli $[44,102,104]$. Conversely, baseline pupil measured during task-related epochs (such as stimulus presentation or the post stimulus decision period) might reflect a combination of autonomic tone and task-relevant factors.

How do phasic and tonic modes of LC activation relate to pupil measures? The terms "phasic" and "tonic" have been proposed to distinguish between different modes of LC activation, not simply between transient and baseline LC activity, respectively [95,96]. Specifically, based primarily on data from rodent studies LC activation patterns are thought to involve: 1) "phasic" mode, which occurs primarily during periods of high task engagement and is dominated by robust, transient responses to task-relevant events that occur when baseline activity is relatively low in the LC; and 2) "tonic" mode, which occurs primarily during periods of high distractibility and involves higher baseline activity and weaker phasic responses in the LC [95,96,103,115]. However, the relevance of these distinct modes of LC activation to conditions that are used commonly to measure pupil diameter in humans, particularly in relation to cognitive processing, is not clear, because these conditions typically involve subjects who are almost always engaged in performing a task and thus likely are in a state akin to "phasic mode." Exceptions to this idea include studies that have used tasks that were designed explicitly to test predictions based on phasic/tonic modes of LC firing [97,98]. Careful use and clear definitions of terminology can help to alleviate some of the confusion that is engendered by the terms "tonic," "phasic," "transient," and "baseline."

How to interpret relationships between baseline and evoked pupil magnitude? A key feature of the proposed phasic/tonic distinction for LC activity is that there is a fundamental trade-off between the magnitude of transient, event-driven activation and baseline levels. Specifically, it has been suggested that maximal transient responses occur at relatively moderate baseline levels (phasic mode) but are attenuated when LC is inactive and arousal is low or when LC is highly active and arousal is high (tonic mode) [96]. This trade-off has been used to interpret pupil measurements, as well, such that transient and baseline values that are inversely related to each other are assumed to reflect a similar relationship in the LC. One challenge to this interpretation is that, as noted above, many task conditions are likely not to involve dramatic changes in baseline arousal state that are needed to evoke the different modes of LC activation. Another challenge is that there is an additional, primary reason for an inverse relationship between transient and baseline pupil measurements that is independent of the LC: the structure of the eye, pupil musculature, and balance between the components of autonomic drive places physical limits on the degree to which the pupil can transiently dilate from a given baseline [54; 99-101]. As a practical consequence, this relationship must be accounted for appropriately (e.g., by quantifying evoked pupil magnitudes in terms of residuals to the evoked-versus-baseline trend [104]) before interpreting the magnitude of an evoked pupil response in terms of a cognitive variable or the magnitude of a related neural response.

How to control variations in baseline pupil? The inherent coupling between baseline and evoked pupil diameter also exacerbates challenges associated with controlling for the effects of luminance on pupil diameter. In particular, changes in the participant's eye or head position or 
the ambient luminance or other visual properties of the experimental set-up can change pupil diameter via the pupillary light reflex. These effects can make it difficult to obtain reliable measures of non-luminance-mediated modulations of baseline pupil diameter that appropriately control for the luminance-mediated modulations, particularly within long experimental sessions or across sessions. These effects can also influence transient pupil responses, the magnitude of which depends on baseline pupil values.

\section{Concluding remarks and future directions}

Pupillometry is a promising, non-invasive technique for measuring changes in brain activity patterns that are associated with particular task-related factors, including arousal, salience, attention, and effort. Compared to other techniques like fMRI, pupillometry is convenient and inexpensive. As with any technique, pupillometry also has its limitations. Pupil size covaries with autonomic as well as cognitive factors, is an indirect marker of neural activity, and has limited temporal resolution for decoding the underlying neural signals, making it challenging to use pupillometry to draw conclusions about specific changes in the brain. The purpose of this review was to describe the strengths and limitations of our current ability to draw such conclusions.

In the first part of this review, we focused on the anatomical circuits that drive pupil changes. We described the $\mathrm{SC}_{\mathrm{i}}$ and LC as two primary hubs for mediating the effects of cognitive factors on the pupil. Several recent studies have used direct measures and manipulations of neural activity in these brain areas to establish a firm anatomical and functional basis for their relationships with pupil diameter. Much more work remains to be done to fully understand how these relationships depend on task conditions, autonomic state, and interactions between the $\mathrm{SC}_{\mathrm{i}}$ and LC and the many other parts of the brain with which they are connected.

In the second part of this review, we addressed current approaches and caveats to interpreting changes in pupil size during goal-directed behavior. Even under constant luminance, pupil size co-varies with a range of behavioral and autonomic states, brain-wide release of neuromodulators, and neural activity in multiple brain regions. In this discussion of the "inverse problem", we argued that these diverse relationships can present serious confounds to the interpretation of task-related pupil dynamics, particularly when the goal is to interpret those dynamics in term of neural activity in a specific brain region or a specific neuromodulatory system. One way to mitigate such confounds is to design tasks that selectively activate these specific regions or systems and then directly test for the expected relationship between neural activation and pupil.

Thus, to make full use of pupillometry as a window into the brain, it will be important to continue to both measure and manipulate activity in the $\mathrm{SC}_{\mathrm{i}}, \mathrm{LC}$, and other brain regions while measuring pupil diameter under well-controlled task conditions. We propose that it will be particularly fruitful to target different neuromodulatory systems in the context of specific cognitive operations that they are thought to mediate, possibly resulting in distinct relationships to pupil diameter. For example, phasic changes in global arousal are thought to be mechanism for rapid reorienting to a behaviorally relevant event. These mechanisms are generally associated with activity in the LC-NE system [52,127-129,148-150]. In contrast, spatially specific attentional changes are more closely associated with activity in the BF-ACh system [57,58]. Well-established tasks can be used to separately manipulate arousal or attention and assess if and how each relates changes in pupil size and activity in these two systems. These studies can then be extended to include measures of neural activity in brain regions that project to or receive projections from these neuromodulatory systems, thereby providing a broad view of the patterns of changes in neural activity throughout the brain that can be inferred from pupillometry. 


\section{HIGHLIGHTS}

- A surge in interest: a search for "pupil" and "behavior" returned a list of $\sim 3$ studies/year from 1967-2008 and >15 studies/year from 2009-2019.

- Studies in non-human primates have identified associations between activity of intermediate layers of the superior colliculus (SCi) and pupil diameter, which are interpreted in terms of SC $_{i}{ }^{\prime} S$ role in processing salient stimuli and the associated orienting responses.

- Studies in humans, non-human primates, and rodents have identified associations between neural activity in the locus coeruleus (LC) and changes in pupil diameter, which are interpreted in terms LC's roles in arousal and cognition.

- Emerging techniques will allow us to: 1) assess neural activity simultaneously in LC, $\mathrm{SC}_{\mathrm{i}}$, and the brain regions with which they are connected; 2) test for causal roles of these circuits in controlling PD; and 3) determine context dependence of these relationships by using carefully controlled behavioral tasks. 


\section{OUTSTANDING QUESTIONS}

1. Do LC and $\mathrm{SC}_{\mathrm{i}}$ have distinct or overlapping roles in controlling pupil size? Is LC modulation of $\mathrm{SC}_{\mathrm{i}}$ responsible, at least in part, for $\mathrm{SC}_{\mathrm{i}}$ control of pupil size?

2. What drives LC neurons, and do different sources of LC drive have different relationships with pupil changes? The two major sources of LC drive are bottom-up autonomic inputs and topdown cortical inputs. How can the effects of these sources be distinguished using measurements of LC and pupil?

3. What is the anatomical and functional basis for LC influence on the EWN? Some tracer studies show projections from LC to EWN, although if and how these contact neurons that are related to pupil control remains unknown.

4. What are the principles that govern context-specific relationships between $\mathrm{SC}_{\mathrm{i}}$ neural activity and pupil size? $\mathrm{SC}_{\mathrm{i}}$ microstimulation evokes pupil dilation. However, under certain conditions SC mean firing rate is negatively correlated with baseline pupil size.

5. What is the anatomical basis for the $\mathrm{SC}_{\mathrm{i}}$-pupil link? In particular, what is the exact role of the $\mathrm{MCN}$, which appears to be ideally positioned to provide a pathway for $\mathrm{SC}_{\mathrm{i}}$ outputs to reach both the sympathetic and parasympathetic arms of the autonomic pupil control circuit?

6. How is activity in different neuromodulatory systems related to changes in pupil size? Direct measurements under limited conditions have been made primarily in the LC-NE system, although there are intriguing findings from studies of the BF-ACh system in mice. We do not know whether and how the dopaminergic and serotonergic neuromodulatory systems are also linked with pupil fluctuations, and whether there might be species-specific differences in these links. 


\section{Box 1: Autonomic circuits that control pupil size: the pupillary light response (PLR)}

In the PLR, light-driven pupil constriction is driven mainly by parasympathetic activation, whereas in the dark response dilation is driven mainly by sympathetic activation (although see below for a more nuanced view). Specifically, changes in light level are conveyed by a class of melanopsin-containing intrinsically photosensitive retinal ganglion neurons that project to the pretectal olivary nucleus (PON) in the midbrain [6-8]. PON neurons project to the EdingerWestphal nucleus (EWN), which is also located in the midbrain [9-11]. A subset of cholinergic EWN neurons provides parasympathetic innervation to the ciliary ganglion (CG), which is located just behind the globe [12-16]. Within the CG, the EWN cholinergic afferents contact neurons that, in turn, project to the eye and innervate the sphincter pupillae, which is a band of muscle encircling the pupil that causes constriction of the pupil when it is contracted $[16,17]$. Sympathetic input to the pupil arises from neurons in the intermediolateral (IML) cell column of the cervical and thoracic spinal cord [18-20]. This input drives the superior cervical ganglion (SCG) that, in turn, innervates directly the dilator pupillae, which is a band of radial muscle that contracts to dilate the pupil $[21,22]$.

In addition to these relatively straightforward parasympathetic and sympathetic pathways to control pupil constriction and dilation, respectively, there are additional autonomic pathways that are not as well understood. For example, pupil constriction might involve not just parasympathetic control but also light-induced inhibition of sympathetic tone, leading to relaxation of the dilator pupillae [23-29]. Similarly, in addition to direct sympathetic activation of the dilator pupillae, withdrawal of parasympathetic tone in low-light levels leads to relaxation of the sphincter pupillae, which also facilitates dilation [23-29]. Moreover, the strength and dynamics of these factors appear to be controlled differently by each component of the autonomic system, with species-specific differences (reviewed in [30-32]). These effects illustrate the complexity of the circuits that control the pupil, even at the lowest levels in the brainstem, and thus the challenges inherent to drawing conclusions about if and how specific circuits are active based solely on observations of the pupil. 
Figure 1. Pathways for pupil control

\section{Pupil constriction}

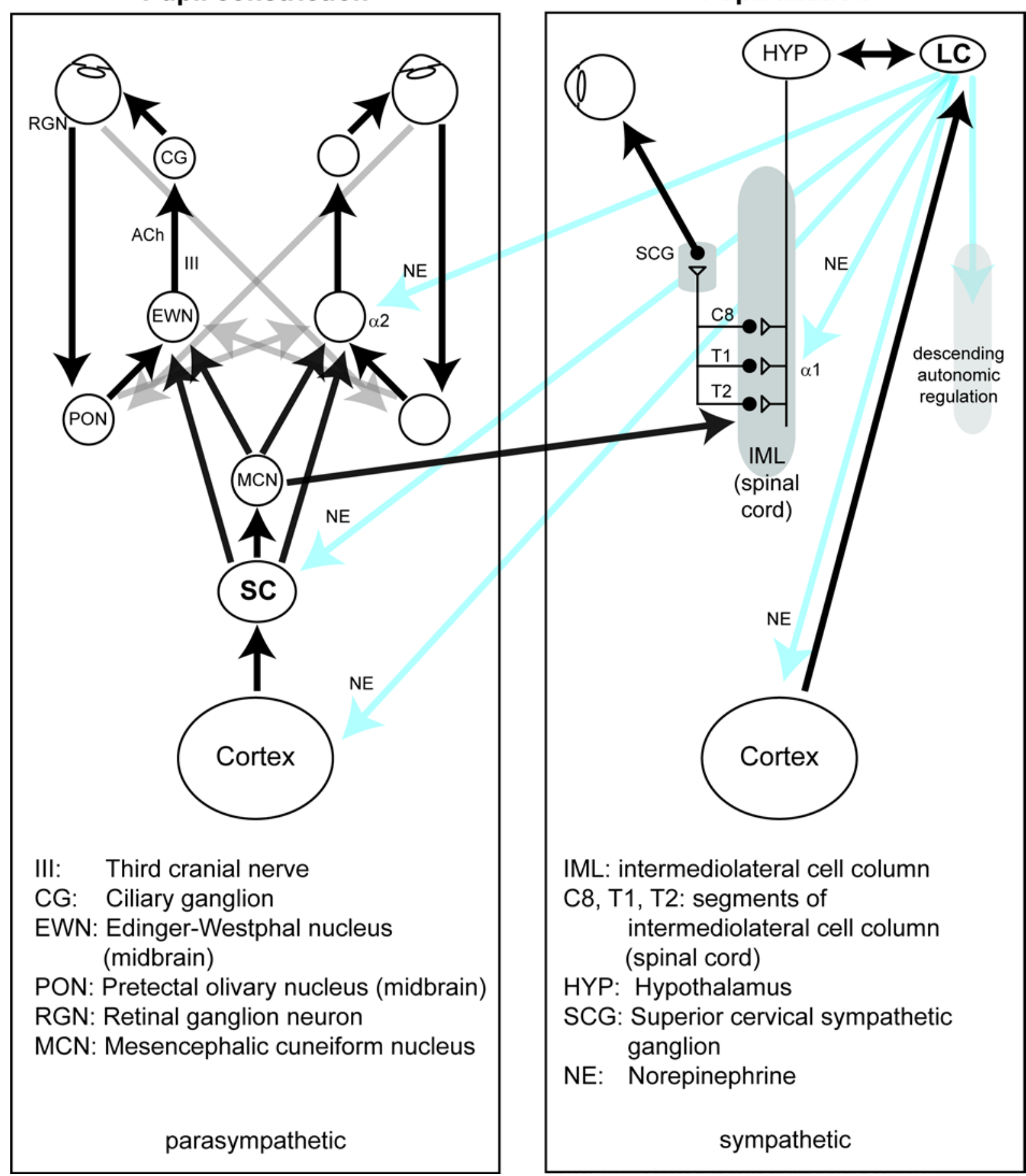

Figure 1. Pathways for control of pupil size. Cortical and subcortical regions involved in pupil dilation (right) and constriction (left). Gray arrows indicate hemisphere-crossing connections at the level of brainstem. Blue arrows indicate LC output to brainstem and cortex. 


\section{Box 2: Pathways for central modulation of autonomic pupil control circuits}

Modulation of pupil control via the superior colliculus (SC). The SC control of pupil likely involves both direct and indirect pathways. The superficial layers of SC $\left(\mathrm{SC}_{\mathrm{S}}\right)$ receive direct retinal ganglion neuron input and project to intermediate layers $\left(\mathrm{SC}_{\mathrm{i}}\right)$. Certain $\mathrm{SC}_{\mathrm{i}}$ neurons then project directly to the EWN and thus can influence the parasympathetic arm of pupil control [4650]. A second, indirect pathway involves $\mathrm{SC}_{\mathrm{i}}$ projections to the mesencephalic cuneiform nucleus (MCN), which, in turn, influences parasympathetic tone via projections to the EWN and sympathetic tone via projections to the thoracic and cervical spinal cord (although the latter projection has to date been shown only in rats) [51]. The strength and nature of these direct and indirect projections in terms of their effect on pupil control circuits are unknown, so there is much work ahead in determining whether and how these pathways might facilitate dilation, constriction, or both [50].

Modulation of pupil control via the locus coeruleus (LC). The LC projects directly to the IML, promoting excitatory sympathetic drive via $\alpha 1$ adrenergic receptors $[31,39,59-62,105]$. The LC also can have indirect sympathetic influence via reciprocal connectivity between the LC and the hypothalamus, which projects to the IML $[31,63]$. Moreover, the LC projects directly to the SC and thus could, in principle, modulate SC-mediated changes in pupil diameter [43]. Finally, the LC is thought to control pupil size via modulation of neural activity in the EWN. The anatomical basis for this modulation remains in debate and may involve direct projections between the LC and EWN, indirect pathways possibly involving common drive via other brainstem nuclei, or both, but is thought to result in inhibition of the EWN, mediated via $\alpha 2$ adrenergic receptors $[64,65]$. In this framework, LC activation should relax parasympathetic drive (by inhibition of EWN) and enhance sympathetic drive (by excitation of $I M L$ ), resulting in pupil dilation [54,63$65,105]$. 


\section{Glossary:}

LC: locus coeruleus

NE: norepinephrine

BF: basal forebrain

ACh: acetylcholine

EEG: electroencephalogram

fMRI: functional magnetic resonance imaging 


\section{REFERENCES}

1. Lowenstein, O., and Loewenfeld, I.E. (1950). Role of sympathetic and parasympathetic systems in reflex dilation of the pupil; pupillographic studies. Arch Neurol Psychiatry. 64:313340.

2. Toates, F.M. (1972). Accommodation function of the human eye. Physiol Rev. 52:828-863.

3. Kuntz, A. (1929). The Autonomic Nervous System (Lea and Febiger, Philadelphia, 1929).

4. Hess, E.H., and Polt, J.M. (1960). Pupil size as related to interest value of visual stimuli. Science. 132:349-350.

5. Hess, E.H., and Polt, J.M. (1964). Pupil Size in Relation to Mental Activity during Simple Problem-Solving. Science. 143:1190-1192.

6. Hattar, S., Liao H.W., Takao M., Berson, D.M., and Yau, K.W. Melanopsin-Containing Retinal. Ganglion Cells: Architecture, Projections, and Intrinsic Photosensitivity. Science. 295:1065-1070.

7. Berson, D.M., Dunn, F.A., and Takao, M. (2002). Phototransduction by Retinal Ganglion Cells That Set the Circadian Clock. Science. 295:1070-1073.

8. Gamlin, P.D., McDougal, D.H., Pokorny, J., Smith, V.C., Yau, K.W., and Dacey, D.M. (2007). Human and Macaque Pupil Responses Driven by Melanopsin-Containing Retinal Ganglion Cells. Vision Res. 47:946-954.

9. Steiger, H.J., and Buttner-Ennever, J.A. (1979). Oculomotor Nucleus Afferents in Monkey Demonstrated with Horseradish-Peroxidase. Brain Research. 160:1-15.

10. Gamlin, P.D., and Clarke, R.J. (1995). The Pupillary Light Reflex Pathway of the Primate. Journal of the American Optometric Association. 66:415-418.

11. Buttner-Ennever, J.A., Cohen, B., Horn, A.K., and Reisine, H. (1996). Pretectal Projections to the Oculomotor Complex of the Monkey and Their Role in Eye Movements. J Comp Neurol. 366:348-359.

12. Strassman, A., Mason, P., Eckenstein, F., Baughman, R.W., and Maciewicz R. (1987). Choline acetyltransferase immunocytochemistry of Edinger-Westphal and ciliary ganglion afferent neurons in the cat. Brain Res. 423:293-304.

13. Horn, A.K., Eberhorn, A., Härtig, W., Ardeleanu, P., Messoudi, A., and Büttner-Ennever, J.A. (2008). Perioculomotor cell groups in monkey and man defined by their histochemical and functional properties: reappraisal of the Edinger-Westphal nucleus. J Comp Neurol. 507:13171335.

14. May, P.J., Warren, S., Gamlin, P.D.R., and Billig, I. (2018). An Anatomic Characterization of the Midbrain Near Response Neurons in the Macaque Monkey. Invest Ophthalmol Vis Sci. 59:1486-1502.

15. Kuchiiwa, S., Kuchiiwa, T, and Suzuki, T. (1989). Comparative anatomy of the accessory ciliary ganglion in mammals. Anatomy and Embryology (Berlin), 180, 199-205.

16. Ruskell, GL. (1990). Accommodation and the Nerve Pathway to the Ciliary Muscle: A Review. Ophthalmic Physiol Opt. 10:239-242.

17. Nomura, T., and Smelser, G.K. (1974). The identification of adrenergic and cholinergic nerve endings in the trabecular meshwork. Invest Ophthalmol. 13:525-532. 
18. Strack, A. M., and Loewy, A. D. (1990). Pseudorabies virus: A highly specific transneuronal cell body marker in the sympathetic nervous system. Journal of Neuroscience, 10, 2139-2147.

19. Hockman, C.H. Essentials of Autonomic Function :The Autonomic Nervous System : Fundamental Concepts from Anatomy, Physiology, Pharmacology, and Neuroscience for Students and Professionals in the Health Sciences. Springfield, Ill., USA: Thomas; 1987.

20. Kardon, R.H. Anatomy and Physiology of the Autonomic Nervous System. In: Miller, NR.; Walsh, FB.; Hoyt, WF., editors. Walsh and Hoyt's Clinical Neuro-Ophthalmology. 6th. Philadelphia: Lippincott Williams \& Wilkins; 2005. p.3v.

21. Mitsuoka, K., Kikutani, T., and Sato, I. (2016). Morphological relationship between the superior cervical ganglion and cervical nerves in Japanese cadaver donors. Brain Behav. 7:e00619.

22. Nagahama, M., Kairada, K., and Oono, S. (1993) Distribution of the innervating neurons of pupillary dilator and tarsal muscles in the superior cervical ganglion. Jpn. J. Ophthalmol. 37:393399.

23. Heller, P.H., Perry, F., Jewett, D.L., and Levine, J.D. (1990). Autonomic components of the human pupillary light reflex. Invest Ophthalmol, Vis. Sci. 31:156-162.

24. Clarke, R.J., Zhang, H., and Gamlin, P.D. (2003). Characteristics of the pupillary light reflex in the alert rhesus monkey. J Neurophysiol. 89:3179-3189.

25. Nisida I, Okada H, and Nakano O. (1960). The Activity of the Ciliospinal Centers and Their Inhibition in Pupillary Light Reflex. Jpn J Physiol. 10:73-84.

26. Passatore, M., and Pettorossi, V.E. (1976) Efferent Fibers in the Cervical Sympathetic Nerve Influenced by Light. Exp Neurol. 52:66-82.

27. Clarke, R.J., and Ikeda, H. Luminance and Darkness Detectors in the Olivary and Posterior Pretectal Nuclei and Their Relationship to the Pupillary Light Reflex in the Rat. I. Studies with Steady Luminance Levels. Exp Brain Res. 1985; 57:224-232.

28. Lowenstein, O., and Loewenfeld, I.E. (1950). Role of sympathetic and parasympathetic systems in reflex dilation of the pupil; pupillographic studies. Pupillographic Studies. Arch. NeurPsych. 64:313-340.

29. Lowenstein, O., and Loewenfeld, I.E. (1950). Mutual role of sympathetic and parasympathetic in shaping of the pupillary reflex to light; pupillographic studies. Arch. Neurol. Psychiatry. 64:341-377.

30. McDougal, D.H., and Gamlin, P.D. (2015). Autonomic control of the eye. Compr Physiol. 5:439-473.

31. Szabadi, E. (2018). Functional Organization of the Sympathetic Pathways Controlling the Pupil: Light-Inhibited and Light-Stimulated Pathways. Front Neurol. 9:1069.

32. Kardon, R. H. (2004). Anatomy and Physiology of the Autonomic Nervous System. In N. Miller, N. Newman (Eds.) Walsh \& Hoyt Clinical Neuro-Ophthalmology, Chapter 20. (6) Williams and Wilkinson.

33. Wurtz, R.H., and Albano, J.E. (1980). Visual-motor function of the primate superior colliculus. Annu Rev Neurosci. 3:189-226.

34. May, P.J. (2006). The mammalian superior colliculus: laminar structure and connections. Prog Brain Res, 151:321-378. 
35. Wurtz, R.H., Joiner, W.M., and Berman, R.A. (2011). Neuronal mechanisms for visual stability: progress and problems. Philos Trans R Soc Lond B Biol Sci. 366:492-503.

36. Arnsten, A.F., and Goldman-Rakic, P.S. (1984). Selective prefrontal cortical projections to the region of the locus coeruleus and raphe nuclei in the rhesus monkey. Brain res 306:9-18

37. Ornstein, K., Milon, H., McRae-Degueurce, A., Alvarez, C., Berger, B., and Würzner, H.P. (1987). Biochemical and radioautographic evidence for dopamine afferents of the locus coeruleus originating in the ventral tegmental area. J. Neural Transm. 70, 183-189.

38. Mizuno, N., and Nakamura, Y. (1970). Direct hypothalamic projections to the locus coeruleus. Brain Res. 19:160-113.

39. Westlund, K.N., and Craig, A.D. (1996). Association of spinal lamina I projections with brainstem catecholamine neurons in the monkey. Exp Brain Res 110:151-162.

40. Price, J.L., and Amaral, D.G. (1981). An autoradiographic study of the projections of the central nucleus of the monkey amygdala. J Neurosci. 1:1242-1259.

41. Morrison, J.H., Foote, S.L., O'Connor, D., and Bloom, F.E. (1982). Laminar, tangential and regional organization of the noradrenergic innervation of the monkey cortex: dopaminebetahydroxylase immunohistochemistry. Brain Res. Bull. 9:309-319

42. Lewis, D.A., Campbell, M.J., Foote, S.L., and Morrison, J.H. (1986).The monoaminergic innervation of primate neocortex. Hum Neurobiol. 5:181-188.

43. Morrison, J.H., and Foote, S.L. (1986). Noradrenergic and serotoninergic innervation of cortical, thalamic, and tectal visual structures in Old and New World monkeys. J Comp Neurol. 243:117-138.

44. Gilzenrat, M.S., Nieuwenhuis, S., Jepma, M., and Cohen, J.D. (2010). Pupil diameter tracks changes in control state predicted by the adaptive gain theory of locus coeruleus function. Cogn. Affect. Behav. Neurosci. 10:252-269.

45. Wang, C.A., and Munoz, D.P. (2015). A circuit for pupil orienting responses: implications for cognitive modulation of pupil size. Curr. Opin. Neurobiol. 33:134-140.

46. Harting, J.K. (1977). Descending pathways from the superior collicullus: an autoradiographic analysis in the rhesus monkey (Macaca mulatta). J. Comp. Neurol. 173:583-612.

47. Huerta, M., and Harting, J. (1984). Connectional organization of the superior colliculus. Trends Neurosci. 7:286-289.

48. Ohtsuka, K., and Sato, A. (1996). Descending projections from the cortical accommodation area in the cat. Invest Ophthalmol Vis. Sci. 37:1429-1436.

49. Konno, S., and Ohtsuka, K. (1997). Accommodation and pupilloconstriction areas in the cat midbrain. Jpn. J. Ophthalmol. 41:43-48.

50. May, P.J., Warren, S., Bohlen, M.O., Barnerssoi, M., and Horn, A.K. (2016). A central mesencephalic reticular formation projection to the Edinger-Westphal nuclei. Brain Struct. Funct. 221:4073-4089.

51. Verberne, A.J. (1995). Cuneiform nucleus stimulation produces activation of medullary sympathoexcitatory neurons in rats. Am. J. Physiol. 268:R752-R758.

52. Nieuwenhuis, S., De Geus, E.J., and Aston-Jones, G. (2011). The anatomical and functional relationship between the $\mathrm{P} 3$ and autonomic components of the orienting response.

Psychophysiology. 48:162-175. 
53. Wang, C.A., Boehnke, S.E., White, B.J., and Munoz, D.P. (2012). Microstimulation of the monkey superior colliculus induces pupil dilation without evoking saccades. J Neurosci. 32:3629-3636.

54. Joshi, S., Li, Y., Kalwani, R.M., and Gold, J.I. (2016). Relationships between Pupil Diameter and Neuronal Activity in the Locus Coeruleus, Colliculi, and Cingulate Cortex. Neuron. 89:221234.

55. Wang, C.A., and Munoz, D.P. (2018). Neural basis of location-specific pupil luminance modulation. Proc. Natl. Acad. Sci. USA. 115:10446-10451.

56. Wang, C.A., Boehnke, S.E., Itti, L., and Munoz, D.P. (2014). Transient pupil response is modulated by contrast-based saliency. J. Neurosci. 34:408-417

57. Thiele, A., and Bellgrove, M.A. (2018). Neuromodulation of Attention. Neuron. 97:769-785.

58. Ma, S., Hangya, B., Leonard, C.S., Wisden, W., and Gundlach, A.L. (2018). Dual-transmitter systems regulating arousal, attention, learning and memory. Neurosci. Biobehav. Rev. 85:2133.

59. Westlund, K.N., and Coulter, J.D. (1980). Descending projections of the locus coeruleus and subcoeruleus/medial parabrachial nuclei in monkey: axonal transport studies and dopaminebeta-hydroxylase immunocytochemistry. Brain Res. 2:235-264.

60. Westlund, K.N., Bowker, R.M., Ziegler, M.G., and Coulter, J.D. (1984). Origins and terminations of descending noradrenergic projections to the spinal cord of monkey. Brain Res. 292:1-16.

61. Clark, F.M., and Proudfitt, H. (1991). The projection of locus coeruleus neurons to the spinal cord in the rat determined by anterograde tracing combined with immunocytochemistry. Brain Res. 538:231-245.

62. Smith, M.S., Schambra, U.B., Wilson, K.H., Page, S.O., and Schwinn, D.A. (1999). Alpha1adrenergic receptors in human spinal cord: specific localized expression of mRNA encoding alpha1-adrenergic receptor subtypes at four distinct levels. Brain Res Mol Brain Res. 63:254261.

63. Nunn, N., Womack, M., Dart, C., and Barrett-Jolley, R. (2011). Function and pharmacology of spinally-projecting sympathetic pre-autonomic neurones in the paraventricular nucleus of the hypothalamus. Curr Neuropharmacol. 9:262-277.

64. Breen, L.A., Burde, R.M., and Loewy, A.D. (1983). Brainstem connections to the EdingerWestphal nucleus of the cat: a retrograde tracer study. Brain Res. 261:303-306.

65. Koss, M.C. (1986). Pupillary Dilation as an Index of Central-Nervous-System Alpha-2Adrenoceptor Activation. Journal of Pharmacological Methods. 15:1-19.

66. Varazzani, C., San-Galli, A., Gilardeau, S., and Bouret, S. (2015). Noradrenaline and dopamine neurons in the reward/effort trade-off: a direct electrophysiological comparison in behaving monkeys. J Neurosci. 35:7866-7877.

67. Reimer, J., McGinley, M.J., Liu, Y., Rodenkirch, C., Wang, Q., McCormick, D.A., and Tolias, A.S. (2016) Pupil fluctuations track rapid changes in adrenergic and cholinergic activity in cortex. Nat Commun. 7:13289.

68. Murphy, P.R., O'Connell, R.G., O'Sullivan, M., Robertson, I.H., and Balsters, J.H. (2014). Pupil diameter covaries with BOLD activity in human locus coeruleus. Hum Brain Mapp. 35:4140-4154. 
69. Murphy, P.R., Robertson, I.H., Balsters, J.H., and O'connell, R.G. (2011). Pupillometry and P3 index the locus coeruleus-noradrenergic arousal function in humans. Psychophysiology. 48:1532-1543.

70. Nieuwenhuis, S., Aston-Jones, G., and Cohen, J. D. (2005). Decision making, the P3, and the locus coeruleus-norepinephrine system. Psychological Bulletin, 131, 510-532.

71. McGinley, M.J., David, S.V., and McCormick, D.A. (2015). Cortical Membrane Potential Signature of Optimal States for Sensory Signal Detection. Neuron. 87:179-192.

72. Reimer, J., Froudarakis, E., Cadwell, C.R., Yatsenko, D., Denfield, G.H., and Tolias, A.S. (2014). Pupil fluctuations track fast switching of cortical states during quiet wakefulness. Neuron. 84:355-362.

73. Vinck, M., Batista-Brito, R., Knoblich, U., and Cardin, J.A. (2015). Arousal and locomotion make distinct contributions to cortical activity patterns and visual encoding. Neuron. 86:740-754.

74. Pickel, V.M., M. Segal, and F.E. Bloom (1974). A radioautographic study of the efferent pathways of the nucleus locus coeruleus. J. Comp. Neurol. 155:15-42.

75. Morrison, J.H., Grzanna, R. Molliver, M.E., and Coyle, J.T. (1978). The distribution and orientation of noradrenergic fibers in the neocortex of the rat: An immunofluorescence study. $\mathrm{J}$. Comp. Neurol. 181:17-40.

76. Waterhouse, B.D., Lin, C.S., Burne, R.A., and Woodward, D.J. (1983). The distribution of neocortical projection neurons in the locus coeruleus. J. Comp. Neurol. 217:418-431.

77. Chandler, D., and Waterhouse, B.D. (2012). Evidence for broad versus segregated projections from cholinergic and noradrenergic nuclei to functionally and anatomically discrete subregions of prefrontal cortex. Front. Behav. Neurosci. 6:20.

78. Chandler, D.J., Lamperski, C.S., and Waterhouse, B.D. (2013). Identification and distribution of projections from monoaminergic and cholinergic nuclei to functionally differentiated subregions of prefrontal cortex. Brain Res. 1522:38-58.

79. Chandler, D.J., Gao, W.J., Waterhouse, B.D. (2014). Heterogeneous organization of the locus coeruleus projections to prefrontal and motor cortices. Proc. Natl. Acad. Sci. USA. 111:6816-6821.

80. Uematsu, A., Tan B.Z., and Johansen, J.P. (2015). Projection specificity in heterogeneous locus coeruleus cell populations: implications for learning and memory. Learn Mem. 22:444-451.

81. Uematsu, A., Tan, B.Z., Ycu, E.A., Cuevas, J.S., Koivumaa, J., Junyent, F., Kremer, E.J., Witten, I.B., Deisseroth, K., and Johansen, J.P. (2017). Modular organization of the brainstem noradrenaline system coordinates opposing learning states. Nat. Neurosci. 20:1602-1611

82. Breton-Provencher, V., and Sur, M. (2018). Active control of arousal by a locus coeruleus GABAergic circuit. Nat Neurosci. 22:218-228.

83. Cerpa, J.C., Marchand, A.R., and Coutureau, E. (2019). Distinct regional patterns in noradrenergic innervation of the rat prefrontal cortex. J Chem Neuroanat. 96:102-109.

84. Kalwani, R.M., Joshi, S., and Gold, J.I. (2014). Phasic activation of individual neurons in the locus ceruleus/subceruleus complex of monkeys reflects rewarded decisions to go but not stop. J. Neurosci. 34:13656-13669.

85. Krnjević, K. (1967). Chemical transmission and cortical arousal. Anesthesiology, 28: 100104. 
86. Jones, B. E. (2004). Activity, modulation and role of basal forebrain cholinergic neurons innervating the cerebral cortex. Prog. Brain Res. 145,157-169.

87. Grossberg, S. (2017). Acetylcholine Neuromodulation in Normal and Abnormal Learning and Memory: Vigilance Control in Waking, Sleep, Autism, Amnesia and Alzheimer's Disease. Front. Neural Circuits. 11:82.

88. Yu AJ, Dayan P. Uncertainty, neuromodulation, and attention. (2005). Neuron. 46:681-692.

89. Van den Brink, R.L., Murphy, P.R., and Nieuwenhuis, S. (2016). Pupil Diameter Tracks Lapses of Attention. PLoS One. 11:e0165274.

90. Binda, P., Pereverzeva, M., Murray, S.O. (2014). Pupil size reflects the focus of featurebased attention. J. Neurophysiol. 112:3046-3052.

91. Unsworth, N., and Robison, M.K. (2016). Pupillary correlates of lapses of sustained attention. Cogn. Affect. Behav. Neurosci. 16:601-615.

92. Nelson, A., and Mooney, R. (2016). The basal forebrain and motor cortex provide convergent yet distinct movement-related inputs to the auditory cortex. Neuron. 90:635-648.

93. Fort, P., Khateb, A., Pegna, A., Muhlethaler, M. and Jones, B.E. (1995). Noradrenergic modulation of cholinergic nucleus basalis neurons demonstrated by in vitro pharmacological and immunohistochemical evidence in the guinea pig brain. Eur. J. Neurosci., 7:1502-1511.

94. Zaborszky, L. and Cullinan, W.E. (1996). Direct catecholaminergic-cholinergic interactions in the basal forebrain. I. Dopamine-beta-hydroxylase- and tyrosine hydroxylase input to cholinergic neurons. J. Comp. Neurol., 374:535-554.

95. Aston-Jones, G., Rajkowski, J., and Cohen, J. (1999). Role of locus coeruleus in attention and behavioral flexibility. Biol Psychiatry 46:1309-1320

96. Aston-Jones, G., and Cohen, J.D. (2005). Adaptive gain and the role of the locus coeruleusnorepinephrine system in optimal performance. J. Comp. Neurol. 493:99-110.

97. Bouret, S., and Richmond, B.J. (2015). Sensitivity of locus ceruleus neurons to reward value for goal-directed actions. J. Neurosci. 35:4005-4014.

98. Hayes, T.R., and Petrov, A.A. (2016). Pupil Diameter Tracks the Exploration-Exploitation Trade-off during Analogical Reasoning and Explains Individual Differences in Fluid Intelligence. J. Cogn. Neurosci. 28:308-318.

99. Semmlow, J., Hansmann, D., and Stark, L. (1975). Variation in pupillomotor responsiveness with mean pupil size. Vision Res. 15:85-90.

100. Sun, F., Tauchi, P., and Stark, L. (1983). Dynamic pupillary response controlled by the pupil size effect. Exp. Neurol. 82:313-324.

101. Steinhauer, S.R., Siegle, G.J., Condray, R., and Pless, M. (2004). Sympathetic and parasympathetic innervation of pupillary dilation during sustained processing. Int. J.

Psychophysiol. 52:77-86.

102. Nassar, M.R., Rumsey, K.M., Wilson, R.C., Parikh, K., Heasly, B., Gold, J.I. (2012). Rational regulation of learning dynamics by pupil-linked arousal systems. Nat. Neurosci. 15:1040-1046.

103. Kane, G.A., Vazey, E.M., Wilson, R.C., Shenhav, A., Daw, N.D., Aston-Jones, G., and Cohen, J.D. (2017). Increased locus coeruleus tonic activity causes disengagement from a patch-foraging task. Cogn. Affect. Behav. Neurosci. 17:1073-1083. 
104. Krishnamurthy, K., Nassar, M.R., Sarode, S., and Gold, J.I. (2017). Arousal-related adjustments of perceptual biases optimize perception in dynamic environments. Nat. Hum. Behav. 1. pii: 0107.

105. Liu, Y., Rodenkirch, C., Moskowitz, N., Schriver, B., and Wang, Q. (2017). Dynamic Lateralization of Pupil Dilation Evoked by Locus Coeruleus Activation Results from Sympathetic, Not Parasympathetic, Contributions. Cell Rep. 20:3099-3112.

106. Jepma, M., and Nieuwenhuis, S. (2011). Pupil diameter predicts changes in the exploration-exploitation trade-off: evidence for the adaptive gain theory. J Cogn Neurosci. 23:1587-1596.

107. Lewis, M.S., Molliver, M.E., Morrison, J.H., and Lidov, H.G. (1979). Complmentarity of dopaminergic and noradrenergic innervation in anterior cingulate cortex of the rat. Brain Res. 164:328-33.

108. Chu, N., and Bloom, F.E. (1973). Norepinephrine-containing neurons: changes in spontaneous discharge patterns during sleeping and waking. Science. 179:908-910.

109. Hong, L., Walz, J.M., and Sajda, P. (2014). Your eyes give you away: prestimulus changes in pupil diameter correlate with poststimulus task-related EEG dynamics. PLoS One. 9:e91321

110. Scharinger, C., Soutschek, A., Schubert, T., and Gerjets, P. (2015). When flanker meets the n-back: What EEG and pupil dilation data reveal about the interplay between the two central-executive working memory functions inhibition and updating. Psychophysiology. 52:1293-1304.

111. Porrino, L.J., and Goldman-Rakic, P.S. (1982). Brainstem innervation of prefrontal and anterior cingulate cortex in the rhesus monkey revealed by retrograde transport of HRP. J. Comp. Neurol. 205:63-76.

112. Pajkossy, P., Szőllősi, Á., Demeter, G., and Racsmány, M. (2017). Tonic noradrenergic activity modulates explorative behavior and attentional set shifting: Evidence from pupillometry and gaze pattern analysis. Psychophysiology. 54:1839-1854.

113. Clewett, D.V., Huang, R., Velasco, R., Lee, T.H., and Mather, M. (2018). Locus Coeruleus Activity Strengthens Prioritized Memories Under Arousal. J. Neurosci. 38:1558-1574.

114. Alnæs, D., Sneve, M.H., Espeseth, T., Endestad, T., van de Pavert, S.H., and Laeng, B. (2014). Pupil size signals mental effort deployed during multiple object tracking and predicts brain activity in the dorsal attention network and the locus coeruleus. J Vis. 14(4).

115. Tervo, D.G.R., Proskurin, M., Manakov, M., Kabra, M., Vollmer, A., Branson, K., and Karpova, A.Y. (2014). Behavioral variability through stochastic choice and its gating by anterior cingulate cortex. Cell. 159:21-32.

116. Larsen, R.S., and Waters, J. (2018). Neuromodulatory Correlates of Pupil Dilation. Front. Neural Circuits. 12:21.

117. Vitiello, B., Martin, A., Hill, J., Mack, C., Molchan, S., and Martinez, R., et al. (1997). Cognitive and behavioral effects of cholinergic, dopaminergic, and serotonergic blockade in humans. Neuropsychopharmacology 16:15-24.

118. Chandler, D.J., Lamperski, C.S., and Waterhouse, B.D. (2013). Identification and distribution of projections from monoaminergic and cholinergic nuclei to functionally differentiated subregions of prefrontal cortex. Brain Res. 1522:38-58.

119. Qiyuan, J., Richer, F.,Wagoner, B. L., and Beatty, J. (1985). The pupil and stimulus probability. Psychophysiology. 22:530-534. 
120. Friedman, D., Hakerem, G., Sutton, S., and Fleiss, J. L. (1973). Effect of stimulus uncertainty on the pupillary dilation response and the vertex evoked potential.

Electroencephalography and Clinical Neurophysiology. 34:475-484.

121. Steinhauer, S. R., and Hakerem, G. (1992). The pupillary response in cognitive psychophysiology and schizophrenia. In D. Friedman \& G. Bruder (Eds.), Psychophysiology and experimental psychopathology: A tribute to Samuel Sutton. Annals of the New York Academy of Sciences. 658:182-204.

122. Bradley, M. M., Miccoli, L., Escrig, M. A., and Lang, P. J. (2008). The pupil as a measure of emotional arousal and autonomic activation. Psychophysiology. 45:602-607.

123. Janisse, M.P. (1977). Pupillometry: The psychology of the pupillary response. Washington, DC: Hemisphere Publishing Co.

124. Matsumoto, M., Matsumoto, K., Abe, H., and Tanaka, K. (2007). Medial prefrontal cell activity signaling prediction errors of action values. Nat. Neurosci. 10:647-656.

125. Hayden, B.Y., Heilbronner, S.R., Pearson, J.M., and Platt, M.L. (2011). Surprise signals in anterior cingulate cortex: neuronal encoding of unsigned reward prediction errors driving adjustment in behavior. J Neurosci. 31:4178-4187.

126. de Gee, J.W., Colizoli, O., Kloosterman, N.A., Knapen, T., Nieuwenhuis, S., and Donner, T.H. (2017). Dynamic modulation of decision biases by brainstem arousal systems. Elife. 6. pii: e23232.

127. Aston-Jones, G., and Bloom, F.E.. (1981) Norepinephrine-containing locus coeruleus neurons in behaving rats exhibit pronounced responses to non-noxious environmental stimuli. $\mathrm{J}$. Neurosci. 1:887-900.

128. Grant, S.J., Aston-Jones, G., and Redmond, D.E.J. (1988). Responses of primate locus coeruleus neurons to simple and complex sensory stimuli. Brain Res. Bull. 21:401-410.

129. Aston-Jones, G., Rajkowski, J., Kubiak, P., and Alexinsky, T. (1994). Locus coeruleus neurons in monkey are selectively activated by attended cues in a vigilance task. J. Neurosci. 14:4467-4480.

130. Lynn, R. Attention, arousal and the orientation reaction. Oxford, UK: Pergamon Press; 1966.

131. Shima, K., and Tanji, J. (1998). Role for cingulate motor area cells in voluntary movement selection based on reward. Science. 282:1335-1338.

132. Wang, C.A., and Munoz, D.P. (2014). Modulation of stimulus contrast on the human pupil orienting response. Eur J Neurosci. 40:2822-2832.

133. Sarafyazd, M., and Jazayeri, M. (2019). Hierarchical reasoning by neural circuits in the frontal cortex. Science. 364: eaav8911.

134. Kahneman, D., and Beatty, J. (1966). Pupil diameter and load on memory. Science. 154:1583-1585.

135. McGuire, J.T., Nassar, M.R., Gold, and J.I., Kable, J.W. (2014). Functionally dissociable influences on learning rate in a dynamic environment. Neuron. 84:870-881.

136. Unsworth N, and Robison, M.K. (2017). A locus coeruleus-norepinephrine account of individual differences in working memory capacity and attention control. Psychon Bull Rev. 24:1282-1311. 
137. Duncan-Johnson, C.C., and Donchin, E. (1977). On quantifying surprise: The variation of event-related potentials with subjective probability. Psychophysiology. 14:456-467.

138. Sommer, M.A., and Wurtz, R.H. (1998). Frontal eye field neurons orthodromically activated from the superior colliculus. J Neurophysiol. 80:3331-3335.

139. Sommer, M.A., and Wurtz, R.H. (2002). A pathway in primate brain for internal monitoring of movements. Science. 296:1480-1482.

140. Berman, R.A., and Wurtz, R.H. (2010). Functional identification of a pulvinar path from superior colliculus to cortical area MT. J Neurosci. 30:6342-6354.

141. Lyon, D.C., Nassi, J.J., and Callaway, E.M. (2010). A disynaptic relay from superior colliculus to dorsal stream visual cortex in macaque monkey. Neuron. 65:270-279.

142. Aston-Jones, G., and Cohen, J.D. (2005). An integrative theory of locus coeruleusnorepinephrine function: adaptive gain and optimal performance. Annu Rev Neurosci. 28:403450.

143. Hayden, B.Y., Heilbronner, S.R., Pearson, J.M., and Platt, M.L. (2011). Surprise signals in anterior cingulate cortex: neuronal encoding of unsigned reward prediction errors driving adjustment in behavior. J Neurosci 31, 4178-4187.

144. O'Reilly, J.X., Schüffelgen, U., Cuell, S.F., Behrens, T.E., Mars, R.B., and Rushworth, M.F. (2013). Dissociable effects of surprise and model update in parietal and anterior cingulate cortex. Proc Natl Acad Sci U S A 110, E3660-E3669.

145. Ebitz, R.B., and Platt, M.L. (2015). Neuronal activity in primate dorsal anterior cingulate cortex signals task conflict and predicts adjustments in pupil-linked arousal. Neuron 85, 628640.

146. Phillips, M. A., Szabadi, E., and Bradshaw, C. M. (2000). Comparison of the effects of clonidine and yohimbine on pupillary diameter at different illumination levels. British Journal of Clinical Pharmacology. 50:65-68.

147. Krauzlis, R.J., Lovejoy, L.P., and Zenon, A. (2013). Superior colliculus and visual spatial attention. Annu. Rev. Neurosci. 36:165-182.

148. Foote, S.L., Berridge, C.W., Adams, L.M., and Pineda, J.A. (1991). Electrophysiological evidence for the involvement of the locus coeruleus in alerting, orienting, and attending. Prog Brain Res. 88:521-352.

149. Witte, E.A., and Marrocco, R.T. (1997) Alteration of brain noradrenergic activity in rhesus monkeys affects the alerting component of covert orienting. Psychopharmacology 132:315-323.

150. Coull, J.T., Nobre, A.C., and Frith, C.D. (2001). The noradrenergic alpha2 agonist clonidine modulates behavioural and neuroanatomical correlates of human attentional orienting and alerting. Cerebral Cortex. 11:73-84. 